\title{
Crystal structure of 5-methyl-4-[1-(5-methyl-4-phenyl-thiazol-2-ylamino)- ethylidene]-2-p-tolyl-2,4-dihydro-pyrazol-3-one, $\mathrm{C}_{23} \mathrm{H}_{22} \mathrm{~N}_{4} \mathrm{OS}$
}

\author{
Amit S. Thakar ${ }^{\mathrm{I}}$, Holger B. Friedrich ${ }^{\mathrm{I}}$, Krishnakant T. Joshi ${ }^{\mathrm{II}}$ and Glenn E. M. Maguire*,

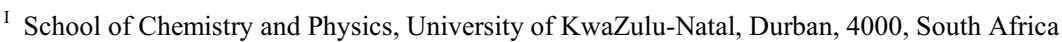 \\ II Department of Chemistry, Navjivan Science College, Dahod, Gujatat 389 151, India
}

Received June 06, 2013, accepted September 18, 2013, available online October 02, 2013, CCDC no. 963864

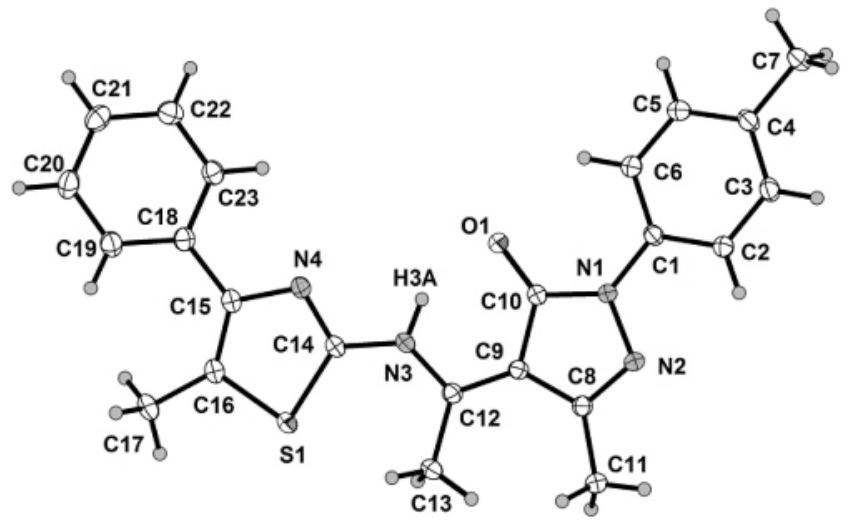

Abstract

$\mathrm{C}_{23} \mathrm{H}_{22} \mathrm{~N}_{4} \mathrm{OS}$, triclinic, $P \overline{1}$ (no. 2),$a=7.1726(9) \AA$,

$b=11.138(2) \AA, c=13.861(2) \AA, \alpha=102.719(6)^{\circ}$,

$\beta=103.317(5)^{\circ}, \gamma=106.645(5)^{\circ}, V=982.6 \AA^{3}, Z=2$,

$R_{\mathrm{gt}}(F)=0.0320, w R_{\text {ref }}\left(F^{2}\right)=0.0892, T=173 \mathrm{~K}$.

Table 1. Data collection and handling.

$\begin{array}{ll}\text { Crystal: } & \text { yellow, block, size } 0.11 \times 0.24 \times 0.48 \mathrm{~mm} \\ \text { Wavelength: } & \text { Mo } K_{\alpha} \text { radiation }(0.71073 \AA) \\ \mu: & 1.87 \mathrm{~cm}^{-1} \\ \text { Diffractometer, scan mode: } & \text { Bruker Smart ApexII CCD, } \varphi \text { and } \omega \\ 2 \theta_{\text {max: }} & 56.66^{\circ} \\ N(h k l)_{\text {measured }}, N(h k l)_{\text {unique }}: & 23645,4827 \\ \text { Criterion for } I_{\text {obs }}, N(h k l)_{\text {gt }}: & I_{\text {obs }}>2 \sigma\left(I_{\mathrm{obs}}\right), 4366 \\ N(\text { param })_{\text {refined }}: & 266 \\ \text { Programs: } & \text { SADABS [18], OLEX2 [19], } \\ & \text { WinGX [20], SHELX [21] }\end{array}$

\section{Source of material}

A solution of 4-acetyl-5-methyl-2-p-tolyl-2,4-dihydro-pyrazol3 -one $(1.00 \mathrm{~g}, 4.3 \mathrm{mmol})$ in methanol $(30 \mathrm{~mL})$ was added to another solution of 5-methyl-4-phenyl-thiazol-2-ylamine $(0.83 \mathrm{~g}$, $4.3 \mathrm{mmol})$ in methanol $(20 \mathrm{~mL})$ under an inert atmosphere. The reaction mixture was refluxed for two hours. Completion of the reaction was monitored by thin layer chromatography (TLC) using hexane/ethyl acetate (7:3). The reaction was allowed to cool to room temperature and then stirred overnight. A yellow precipitate formed which was then filtered and washed with methanol $(10 \mathrm{~mL})$. The crude product was purified by crystallization from ethanol yielding brown crystals. (1.38 g, 79\% yield). M.p. $=495$ 496 K. Crystals suitable for X-ray diffraction were obtained by slow evaporation of a solution of the title compound in a mixture of dichloromethane and hexane at room temperature.

\section{Experimental details}

All $\mathrm{H}$ atoms were positioned geometrically and allowed to ride on their respective parent atoms. All $\mathrm{H}$ atoms were refined isotropically. The absorpton correction was based on fitting a function to the empirical transmission surface as sampled by multiple equivalent measurements [18].

\section{Discussion}

Schiff base ligands have been employed in a diverse range of applications. These areas include biochemical mimics [1] and coordination chemistry $[2,3]$. This family of ligands can coordinate to metals anywhere from a monodentate [4] to a multidentate [5] arrangement. More recently Schiff base metal complexes have been studied for use as antibacterial $[6,7]$, antifungal $[8,9]$, antitumor drugs $[10,11]$ as well as homogenous catalysts $[12,13]$. We previously have reported the synthesis of the title compound and from solution NMR deduced that this Schiff base is present as the keto-imine tautomeric form [14]. In this report we demonstrate that this is also the dominant isomer in the solid phase. This phenomenon has been reported for derivatives of the same general family of molecules [15-17]. The diffraction data revealed an

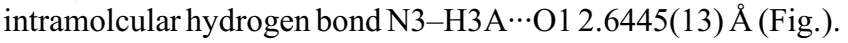

Table 2. Atomic coordinates and displacement parameters (in $\AA^{2}$ ).

\begin{tabular}{llrrrl}
\hline Atom & Site & \multicolumn{1}{l}{$y$} & \multicolumn{1}{c}{$z$} & $U_{\text {iso }}$ \\
\hline $\mathrm{H}(2)$ & $2 i$ & 0.1324 & -0.4114 & 0.0691 & 0.021 \\
$\mathrm{H}(3)$ & $2 i$ & 0.1437 & -0.4062 & -0.0969 & 0.023 \\
$\mathrm{H}(5)$ & $2 i$ & 0.2989 & -0.0116 & -0.0042 & 0.030 \\
$\mathrm{H}(6)$ & $2 i$ & 0.2888 & -0.0138 & 0.1624 & 0.029 \\
$\mathrm{H}(7 \mathrm{~A})$ & $2 i$ & 0.0931 & -0.2207 & -0.2199 & 0.036 \\
$\mathrm{H}(7 \mathrm{~B})$ & $2 i$ & 0.2793 & -0.2741 & -0.2056 & 0.036 \\
$\mathrm{H}(7 \mathrm{C})$ & $2 i$ & 0.3244 & -0.1190 & -0.1710 & 0.036 \\
$\mathrm{H}(11 \mathrm{~A})$ & $2 i$ & 0.0628 & -0.5175 & 0.3290 & 0.029 \\
$\mathrm{H}(11 \mathrm{~B})$ & $2 i$ & -0.0438 & -0.4473 & 0.4017 & 0.029 \\
$\mathrm{H}(11 \mathrm{C})$ & $2 i$ & 0.1942 & -0.4253 & 0.4454 & 0.029 \\
$\mathrm{H}(13 \mathrm{~A})$ & $2 i$ & 0.2761 & -0.1418 & 0.6382 & 0.032 \\
$\mathrm{H}(13 \mathrm{~B})$ & $2 i$ & 0.1138 & -0.2707 & 0.5480 & 0.032 \\
$\mathrm{H}(13 \mathrm{C})$ & $2 i$ & 0.0425 & -0.1533 & 0.5953 & 0.032 \\
$\mathrm{H}(17 \mathrm{~A})$ & $2 i$ & 0.4495 & 0.3465 & 0.9324 & 0.031 \\
$\mathrm{H}(17 \mathrm{~B})$ & $2 i$ & 0.2112 & 0.2586 & 0.8958 & 0.031 \\
$\mathrm{H}(17 \mathrm{C})$ & $2 i$ & 0.2808 & 0.4034 & 0.8844 & 0.031 \\
$\mathrm{H}(19)$ & $2 i$ & 0.6065 & 0.5304 & 0.8776 & 0.026 \\
$\mathrm{H}(20)$ & $2 i$ & 0.7669 & 0.7525 & 0.8972 & 0.029 \\
$\mathrm{H}(21)$ & $2 i$ & 0.7399 & 0.8278 & 0.7514 & 0.029 \\
$\mathrm{H}(22)$ & $2 i$ & 0.5450 & 0.6794 & 0.5852 & 0.026 \\
$\mathrm{H}(23)$ & $2 i$ & 0.3851 & 0.4571 & 0.5650 & 0.023 \\
$\mathrm{H}(3 \mathrm{~A})$ & $2 i$ & 0.2870 & 0.0491 & 0.4665 & 0.020 \\
& & & & & \\
\hline & & & & &
\end{tabular}

\footnotetext{
* Correspondence author (e-mail: maguireg@ukzn.ac.za)
} 
Table 3. Atomic coordinates and displacement parameters (in $\left.\AA^{2}\right)$.

\begin{tabular}{|c|c|c|c|c|c|c|c|c|c|c|}
\hline Atom & Site & $x$ & $y$ & $z$ & $U_{11}$ & $U_{22}$ & $U_{33}$ & $U_{12}$ & $U_{13}$ & $U_{23}$ \\
\hline $\mathrm{C}(1)$ & $2 i$ & $0.2107(2)$ & $-0.2129(1)$ & $0.13260(8)$ & $0.0165(5)$ & $0.0173(5)$ & $0.0127(5)$ & $0.0052(4)$ & $0.0051(4)$ & $0.0043(4)$ \\
\hline$C(2)$ & $2 i$ & $0.1670(2)$ & $-0.3297(1)$ & $0.05470(8)$ & $0.0211(5)$ & $0.0158(5)$ & $0.0158(5)$ & $0.0067(4)$ & $0.0059(4)$ & $0.0047(4)$ \\
\hline$C(3)$ & $2 i$ & $0.1741(2)$ & $-0.3262(1)$ & $-0.04431(9)$ & $0.0225(5)$ & $0.0190(5)$ & $0.0146(5)$ & $0.0077(4)$ & $0.0059(4)$ & $0.0025(4)$ \\
\hline$C(4)$ & $2 i$ & $0.2247(2)$ & $-0.2082(1)$ & $-0.06805(8)$ & $0.0198(5)$ & $0.0227(6)$ & $0.0138(5)$ & $0.0058(4)$ & $0.0058(4)$ & $0.0058(4)$ \\
\hline$C(5)$ & $2 i$ & $0.2658(2)$ & $-0.0931(1)$ & $0.01057(9)$ & $0.0385(7)$ & $0.0174(6)$ & $0.0196(6)$ & $0.0059(5)$ & $0.0118(5)$ & $0.0076(4)$ \\
\hline$C(6)$ & $2 i$ & $0.2597(2)$ & $-0.0938(1)$ & $0.11012(9)$ & $0.0380(7)$ & $0.0152(5)$ & $0.0179(5)$ & $0.0059(5)$ & $0.0113(5)$ & $0.0039(4)$ \\
\hline $\mathrm{C}(8)$ & $2 i$ & $0.1366(2)$ & $-0.3211(1)$ & $0.34484(8)$ & $0.0141(4)$ & $0.0153(5)$ & $0.0147(5)$ & $0.0046(4)$ & $0.0037(4)$ & $0.0046(4)$ \\
\hline $\mathrm{C}(9)$ & $2 i$ & $0.1955(2)$ & $-0.1822(1)$ & $0.39771(8)$ & $0.0158(5)$ & $0.0152(5)$ & $0.0139(5)$ & $0.0049(4)$ & $0.0046(4)$ & $0.0058(4)$ \\
\hline$C(10)$ & $2 i$ & $0.2419(2)$ & $-0.1172(1)$ & $0.32120(8)$ & $0.0159(5)$ & $0.0151(5)$ & $0.0132(5)$ & $0.0053(4)$ & $0.0045(4)$ & $0.0039(4)$ \\
\hline $\mathrm{C}(11)$ & $2 i$ & $0.0828(2)$ & $-0.4379(1)$ & $0.38359(9)$ & $0.0235(5)$ & $0.0156(5)$ & $0.0179(5)$ & $0.0052(4)$ & $0.0061(4)$ & $0.0064(4)$ \\
\hline $\mathrm{C}(12)$ & $2 i$ & $0.2049(2)$ & $-0.1125(1)$ & $0.49607(8)$ & $0.0153(5)$ & $0.0160(5)$ & $0.0139(5)$ & $0.0053(4)$ & $0.0036(4)$ & $0.0050(4)$ \\
\hline $\mathrm{C}(13)$ & $2 i$ & $0.1550(2)$ & $-0.1749(1)$ & $0.57630(9)$ & $0.0309(6)$ & $0.0191(6)$ & $0.0156(5)$ & $0.0078(5)$ & $0.0101(4)$ & $0.0070(4)$ \\
\hline $\mathrm{C}(14)$ & $2 i$ & $0.2911(2)$ & $0.1191(1)$ & $0.60720(8)$ & $0.0162(5)$ & $0.0179(5)$ & $0.0124(5)$ & $0.0068(4)$ & $0.0046(4)$ & $0.0043(4)$ \\
\hline$C(15)$ & $2 i$ & $0.3861(2)$ & $0.3270(1)$ & $0.70363(8)$ & $0.0156(5)$ & $0.0179(5)$ & $0.0141(5)$ & $0.0073(4)$ & $0.0036(4)$ & $0.0030(4)$ \\
\hline$C(17)$ & $2 i$ & $0.3158(2)$ & $0.3240(1)$ & $0.88094(9)$ & $0.0219(5)$ & $0.0261(6)$ & $0.0142(5)$ & $0.0098(4)$ & $0.0067(4)$ & $0.0033(4)$ \\
\hline $\mathrm{C}(18)$ & $2 i$ & $0.4789(2)$ & $0.4699(1)$ & $0.71918(9)$ & $0.0178(5)$ & $0.0175(5)$ & $0.0177(5)$ & $0.0085(4)$ & $0.0065(4)$ & $0.0038(4)$ \\
\hline$C(19)$ & $2 i$ & $0.5939(2)$ & $0.5600(1)$ & $0.81800(9)$ & $0.0247(6)$ & $0.0206(6)$ & $0.0176(5)$ & $0.0099(4)$ & $0.0039(4)$ & $0.0043(4)$ \\
\hline $\mathrm{C}(20)$ & $2 i$ & $0.6897(2)$ & $0.6925(1)$ & $0.8297(1)$ & $0.0274(6)$ & $0.0183(6)$ & $0.0225(6)$ & $0.0087(5)$ & $0.0036(5)$ & $0.0002(4)$ \\
\hline $\mathrm{C}(21)$ & $2 i$ & $0.6732(2)$ & $0.7375(1)$ & $0.7432(1)$ & $0.0259(6)$ & $0.0168(6)$ & $0.0300(6)$ & $0.0094(5)$ & $0.0100(5)$ & $0.0057(5)$ \\
\hline $\mathrm{C}(22)$ & $2 i$ & $0.5582(2)$ & $0.6491(1)$ & $0.6445(1)$ & $0.0261(6)$ & $0.0223(6)$ & $0.0235(6)$ & $0.0122(5)$ & $0.0112(5)$ & $0.0095(5)$ \\
\hline$C(23)$ & $2 i$ & $0.4625(2)$ & $0.5167(1)$ & $0.63259(9)$ & $0.0208(5)$ & $0.0205(6)$ & $0.0175(5)$ & $0.0087(4)$ & $0.0068(4)$ & $0.0043(4)$ \\
\hline $\mathrm{N}(1)$ & $2 i$ & $0.2012(1)$ & $-0.21830(9)$ & $0.23264(7)$ & $0.0200(4)$ & $0.0126(4)$ & $0.0137(4)$ & $0.0044(3)$ & $0.0058(3)$ & $0.0044(3)$ \\
\hline $\mathrm{N}(2)$ & $2 i$ & $0.1385(1)$ & $-0.34206(9)$ & $0.24842(7)$ & $0.0180(4)$ & $0.0130(4)$ & $0.0161(4)$ & $0.0044(3)$ & $0.0052(3)$ & $0.0056(3)$ \\
\hline $\mathrm{N}(3)$ & $2 i$ & $0.2628(1)$ & $0.02012(9)$ & $0.51817(7)$ & $0.0207(4)$ & $0.0166(5)$ & $0.0120(4)$ & $0.0060(3)$ & $0.0062(3)$ & $0.0043(3)$ \\
\hline $\mathrm{N}(4)$ & $2 i$ & $0.3627(1)$ & $0.24127(9)$ & $0.60835(7)$ & $0.0179(4)$ & $0.0171(5)$ & $0.0138(4)$ & $0.0068(3)$ & $0.0050(3)$ & $0.0035(3)$ \\
\hline $\mathrm{O}(1)$ & $2 i$ & $0.3058(1)$ & $0.00358(8)$ & $0.33180(6)$ & $0.0249(4)$ & $0.0130(4)$ & $0.0155(4)$ & $0.0045(3)$ & $0.0072(3)$ & $0.0043(3)$ \\
\hline$S(1)$ & $2 i$ & $0.24113(4)$ & $0.09854(3)$ & $0.72046(2)$ & $0.0197(1)$ & $0.0183(1)$ & $0.0129(1)$ & $0.0067(1)$ & $0.0062(1)$ & $0.0048(1)$ \\
\hline
\end{tabular}

Acknowledgments. The authors wish to thank Dr Bernard Owaga from the University of KwaZulu-Natal for assistance with the data collection and refinement.

\section{References}

1. Lozier, R. H.; Bogomolni, R. A.; Stoeckenius, W.: Bacteriorhodopsin: a light-driven proton pump in Halobacterium Halobium. Biophys. J. 15 (1975) 955-962.

2. Kargar, H.; Jamshidvand, A.; Fun, H. K.; Kia, R.: 6,6'-Dieth-oxy-2,2'[2,2-dimethyl-propane-1,3-diylbis(nitrilo-methylidyne)]diphenolatonickel(II) monohydrate. Acta Crystallogr. E65 (2009) m403-m404.

3. Yeap, C. S.; Kia, R.; Kargar H.; Fun, H. K.: 6,6'-Dimethoxy-2,2'-[2,2dimethylpropane-1,3-diylbis(nitrilomethylidyne)]diphenolato nickel(II) 1.78-hydrate. Acta Crystallogr. E65 (2009) m570-m571.

4. Tian, Y.P.; Duan, C. Y.; Zhao, C. Y.; You, X. Z.; Mak, T. C. W.; Zhang, Z. Y.: Synthesis, crystal structure, and second-order optical nonlinearity of bis(2-chlorobenzaldehyde thiosemicarbazone)cadmium halides $\left(\mathrm{CdL}_{2} \mathrm{X}_{2}: \mathrm{X}=\mathrm{Br}, \mathrm{I}\right)$. Inorg. Chem. 36 (1997) 1247-1252.

5. Bodige, S. G.; Rogers, R. D.; Blackstock, S. C.: Supramolecular networks via pyridine $\mathrm{N}$-oxide $\mathrm{CH} \cdots \mathrm{O}$ hydrogen bonding in the crystal structures of 2,2'-dithiobis (pyridine $N$-oxide) and its complexes with 1,2,4,5tetracyanobenzene and pyromellitic dianhydride. Chem. Commun. (1997) 1669-1670.

6. Mehta, B. H.; Salunke, A. S.: Spectral, Thermal and biological studies of $\mathrm{Mn}(\mathrm{II})$ and $\mathrm{Fe}(\mathrm{II})$ complexes of Schiff base derived from fat $p$ dimethylaminobenzaldehyde and anthranilic acid. Asian J. Chem. 18 (2006) 1326-1334.

7. Singha, R. V.; Biyalaa, M. K.: Structural studies with antimicrobial and antifertility activity of a monofunctional bidentate ligand with its boron(III), palladium(II), and platinum(II) complexes. Phosphorus Sulfur. 181 (2006) 1477-1491.

8. Chohan,Z.H.; Arif, M.; Shafiq, Z.; Yaqub, M.; Supuran, C. T.: In vitro antibacterial, antifungal \& cytotoxic activity of some isonicotinoylhydrazide Schiff's bases and their cobalt (II), copper (II), nickel (II) and zinc (II)complexes. J. Enzym. Inhib. Med. Ch. 21 (2006) 95-103.

9. Parekha, H. M.; Panchala, P. K.; Patela M. N.: In vitro fungitoxic activity of some Schiff bases and their oxovanadium(IV) complexes. Toxicol. Environ. Chem. 88 (2006) 579-586.
10. Adsule, S.; Barve, V.; Chen, D.; Ahmed, F.; Dou, Q. P.; Padhye, S.; Sarkar, F. H.: Novel Schiff base copper complexes of quinoline-2 carboxaldehyde as proteasome inhibitors in human prostate. Cancer Cells. J. Med. Chem. 49 (2006) 7242-7246.

11. Zhonga, X.; Yib, J.; Sunb, J.; Weib, H. L.; Liua, W. S.; Yuc, K. B.: Synthesis and crystal structure of some transition metal complexes with a novel bis-Schiff base ligand and their antitumor activities. Eur. J. Med. Chem. 41 (2006) 1090-1092.

12. Handa, S.; Gnanadesikan, V.; Matsunaga, S.; Shibasaki, M.: Syn-selective catalytic asymmetric nitro-Mannich reactions using a heterobimetallic Cu-Sm-Schiff base complex. J. Am. Chem. Soc. 129 (2007) 4900-4901.

13. Salavati-Niasari, M.; Shaterian, M.; Ganjali, M. R.; Norouzi, P.: Oxidation of cyclohexene with tert-butylhydroperoxide catalysted by host (nanocavity of zeolite-Y)/guest (Mn(II), $\mathrm{Co}(\mathrm{II}), \mathrm{Ni}(\mathrm{II})$ and $\mathrm{Cu}(\mathrm{II}) \mathrm{com}$ plexes of $N, N^{\prime}$-bis(salicylidene)phenylene-1,3-diamine) nanocomposite materials (HGNM). J. Mol. Catal. A-Chem. 261 (2007) 147-155.

14. Joshi, K. T.; Pancholi, A. M.; Pandya, K. S.; Thakar, A. S.: Synthesis, characterization and antibacterial activity of novel Schiff base derived from 4-acetyl-3-methyl-1-(4'-methyl-phenyl)-2-pyrazolin-5-one and its transition metal complexes. Int. J. Res. Chem. Environ. 1 (2011) 63-69.

15. Liu, L.; Jia, D-Z.; Ji, Y-L.; Yu, K-B.: Synthesis, structure and photochromic propertiesof 4-acyl pyrazolone derivants. J. Photochem. Photobiol. A154 (2003) 117-122.

16. Jadeja, R.N.; Shah, J.R.; Suresh, E.; Paul, P.: Synthesis and structural characterization of some Schiff bases derived from 4-[(aryl)imino-ethyl]3-methyl-1-(4-methylphenyl)-2-pyrazolin-5-one and spectroscopic studies of their Cu(II) complexes. Polyhedron 23 (2004) 2465-2474.

17. Jadeja, R.N.; Shah, J.R.: Studies on some oxovanadium(IV) complexes of acyl pyrazolone analogues. Polyhedron 26 (2007) 1677-1685.

18. APEX2, SAINT and SADABS, Bruker AXS Inc., (2006) Madison, Wisconsin, USA.

19. Dolomanov, O. V.; Bourhis, L. J.; Gildea, R. J.; Howard, J. A. K; Puschmann, H.: OLEX2: A complete structure solution, refinement \& analysis program. J. Appl. Crystallogr. 42 (2009) 339-341.

20. Farrugia, L. J.: WinGX and ORTEP for Windows: an update. J. Appl. Crystallogr. 45 (2012) 849-854.

21. Sheldrick, G. M.: A short history of SHELX. Acta Crystallogr. A64 (2008) 112-122. 